\title{
ADAPTIVE TRAFFIC CONTROL SYSTEM
}

\author{
Prof. M. A. Pardesi \\ Professor, Department of Computer Science and Engineering, D. Y. Patil College of Engineering \&Technology, \\ Kolhapur, Maharashtra, India
}

\author{
Saquib S. Aowte, Tanmay V. Kakare, Revati U. Bhosale, Swayambhoo R. Patil, \\ Department of Computer Science and Engineering, D. Y. Patil College of Engineering \&Technology, Kolhapur, \\ Maharashtra, India
}

\begin{abstract}
This paper proposes an innovative method to change the timing delay of the traffic light systems automatically according to the traffic density. Nowadays traffic congestion has become a subject of concern at the global as well as local level. Existing traffic signaling system is based on fixed time delay which may incompetent if one lane is operational rather than others. This leads to increase in excessive time delay of the traffic. Sometimes high traffic congestion at one side of a lane needs longer green signal as compared to existing system it analyses the traffic density of each lane and accordingly varies the time period of the signal at that time. To optimize this problem we have to design adaptive traffic control system (ATCS). This can be achieved by using magnetic loop vehicle detection sensor which detects the presence of vehicle. Once the presence of vehicle is detected the time period of signal is adjusted with the help of microcontroller (ATMEGA 328P). The sensor which are grooved inside the road at each lane of junction which will detect the presence of vehicle passing that lane and sends the information to microcontroller. Based on the collected information it will decide which lane should get additional time and which lane's time should be deducted. This will happen only once in a single loop.
\end{abstract}

Keywords- Magnetic loop vehicle detection sensor, Microcontroller, Traffic light system, LED lights, LCD.

\section{INTRODUCTION}

The traffic scenario in India and also at global level, since high need of transportation and increase in number of vehicles, has become a topic of concern. As the number of vehicles running on road are increasing continuously, the infrastructure of traffic regulation system must be updated. There are many factors that lead to traffic congestion such as density of vehicles on the roads, human habits, social behaviors and traffic light system. One major factor is due to traffic light system that controls traffic at junction. No doubt traffic police are there to regulate traffic congestion, but what if there is an automated system which regulates the traffic. The problem specific to Indian cities is lane management. We would definitely assume that in order to accomplish traffic management, individual lanes have to be better managed. But the primary problem is overlooked is the dynamic growth of vehicles i.e. the accumulation of these vehicles at various cross roads and junctions. Another problem faced in developing cities is the amount of green signal time given to the empty streets, this signal time can be divided among the streets having vehicles and traffic density can be decreased. The system is based on internet of things(IOT) technology. IOT connects physical devices to the internet and allows to the exchange data. The objective of paper is to create the innovative way to reduce the traffic congestion. The proposed system uses magnetic loop vehicle detection sensor and these sensors are grooved at the beginning of each lane which will detect the presence of vehicles in that particular magnetic loop. The sensed data will be provided as a input to the IOT device which includes microcontroller. Based on the data collected by the microcontroller about the existence of vehicle in that specific loop, the timer of traffic light system will be adjusted. Microcontroller and signaling system communicates with the help of TCP/IP protocol. Additionally, it includes ESP8266 which is low cost WIFI microchip with full TCP/IP stack and microcontroller capability. LCD display is used to display the timer of each lane i.e. Lane1, Lane2, Lane3, Lane4 respectively. Web application is developed by using REST API which monitors the live scenario of traffic. In case of any emergency, the system administrator can manually alter the signal (Example: Red to green light or Vice-versa).

\section{EXISTING SYSTEM}

The traffic scenario is increasing day by day in the metropolitan cities due to high need of transportation. As a result, this scenarios leads to high traffic jam in the metro cities. In the present circumstances, the traffic control system is performed by two standard traffic control systems such as Manual controlling which requires manpower to control the traffic [1] and Automatic traffic controlling system which includes timers and electronic sensors[2]. Figure 2.1 shows traffic congestion. 


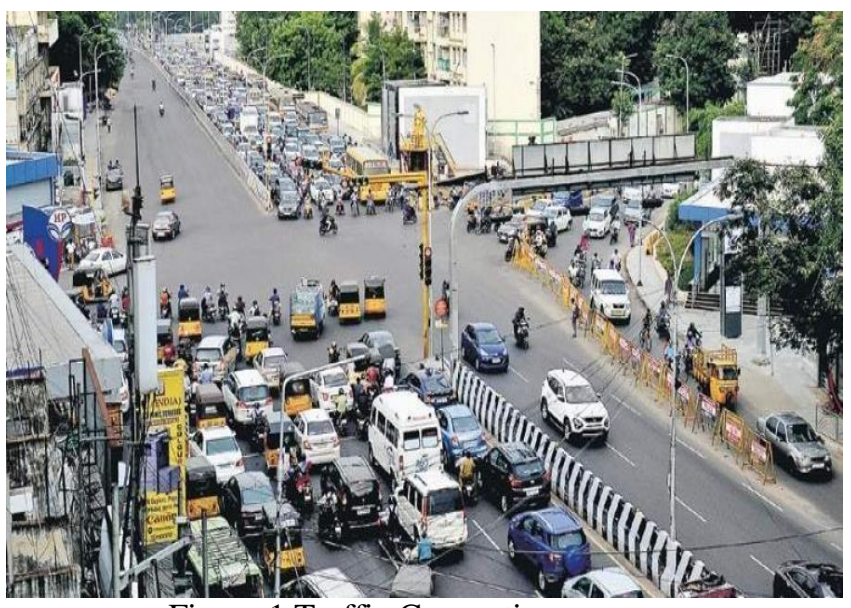

Figure 1 Traffic Congestion

\section{PROPOSED SYSTEM}

This model works on the principle of changing time delay of traffic signals based on the number of vehicles passing through the assigned section of the road. Instead of using fixed time delay for each lane, we are using magnetic loop vehicle detection sensor on each lane of the junction. Whenever there is presence of vehicle in the particular loop, the sensor will detect the metal and sends information to microcontroller. There are six cases according to which the system will work: The first case is considered as the best case where all the 4 lanes have high density. In such case every lane will be provided with equal time. The second case states that the density of opposite lane is higher. Here due to the low density of 2 lanes the time which was not used by the lower density lane's will be added to the lanes with high density. The third case has a single lane with high density and other 3 lanes with low density. The fourth case consist of consecutive high and consecutive low density. Here in the loop, the unoccupied time of previous lane will be added to the next lane having high density. The fifth case consist of consecutive low density. Similar to the fourth case the unoccupied time of previous lane will be added to the next lane which having high density.

\section{REQUIREMENTS}

Following would be the technical requirements to develop system:

\section{Software:}

- Operating System- Windows 7,8 or 10

- mikroC PRO for AVR

- $\quad$ SinaProg 2.1
- PADS Logic, Layout

- Language- Embedded c

\section{Hardware:}

- Magnetic loop vehicle detection sensor

- AtMega328P microcontroller

- 16x2 LCD Screen

- LED Lights

- Transformer

\section{RESULTS}

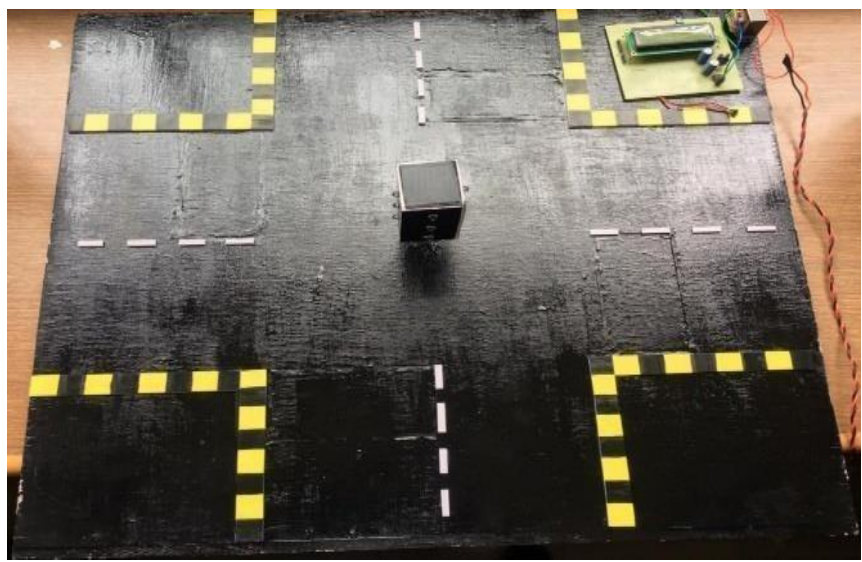

Figure 2 shows the Prototype Designing

Above figure shows the prototype design of the proposed system consist of 4 lane and each lane has a sensor which grooved within the board, signal placed at the center.

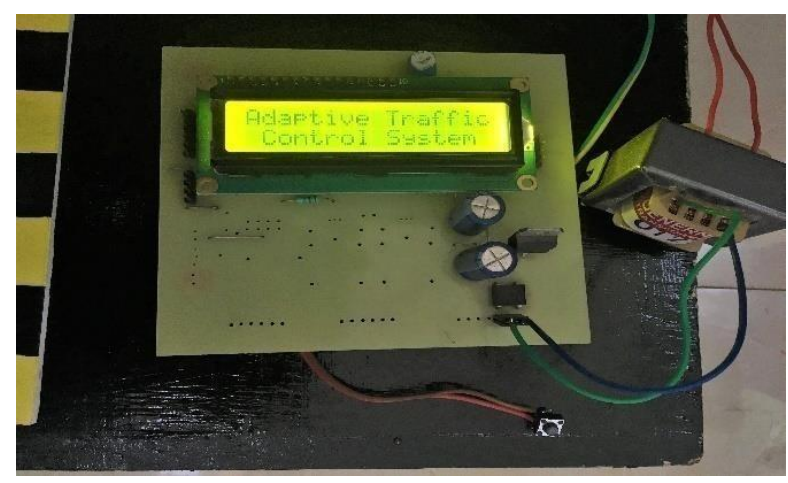

Figure 3 shows LCD and signal interfacing

Above figure shows the PCB which consist of 16/2 LCD display, regulator, IC, capacitor, programming port, microcontroller port, transformer, reset pot, resistors. 


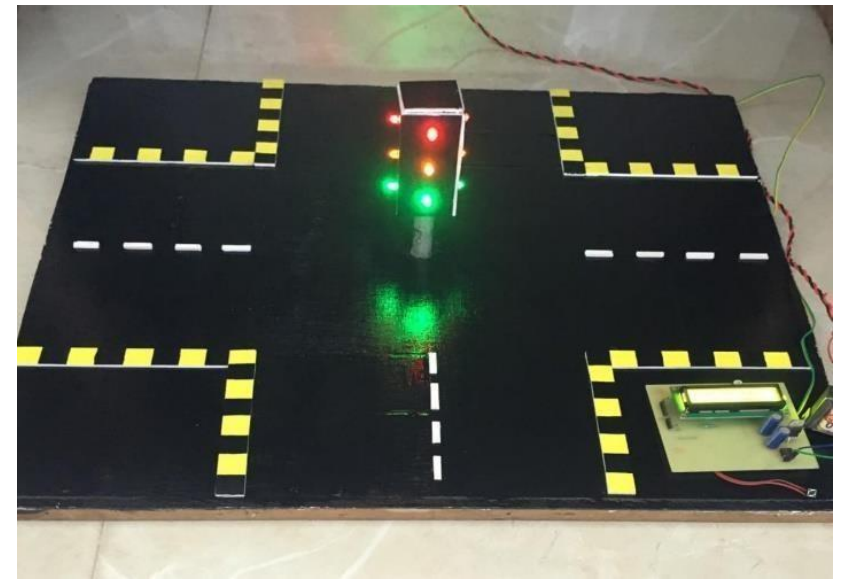

Fig. 4. LCD Interface

Above figure shows the LCD which is interfaced on the prototype and all LED's of signal in ON state.

\section{FUTURE WORK}

1) Traffic Density Module: This module consists of hardware such as sensor which will be used to detect traffic density (Presence of vehicle). The sensor forwards that information to microcontroller. The microcontroller is programmed in such a way that the data which is collected by the sensor, microcontroller will adjust the timer of the signal.

2) Connectivity Module: This module works as a interface between hardware module and software that is microcontroller and web application. The output from the microcontroller will be considered as input to this connectivity module. This data will be given further to the web application to monitor live scenario of traffic congestion.

3) Web Application Module: In this module, admin will login to web portal. The web application will be used for continuously monitoring the status of the system which means it will show which lane at the junction having the high traffic density. It will also display the lane to which the additional time is added. The application will also be used to manually ON/OFF system in working hours, to manually alter the signal light with red to green or green to red if any emergency is occurred.

\section{CONCLUSION}

As the traffic scenario is increasing day by day due to high need of transportation. The system of traffic control system needs to be updated. Adaptive Traffic Control System is aiming to reduce traffic congestion, to minimize the negative effects of traffic towards the environment and people with the perspective of traffic management.

\section{REFERENCE}

1. Chandramohan, J., Nagarajan, R., Satheeshkumar, K., Ajithkumar,N., Gopinath, P. A., \& Ranjithkumar, S. (2017). "Intelligent smart home automation and security system using Arduino and Wi-fi." International Journal of Engineering and Computer Science,6(3)

2. Priyadharshni K., Manikandan k. S. (2019). "Automatic Traffic Control System Based on the Vehicular Density". International Journal of Engineering and Technology (IRJET).

3. https://ieeexplore.ieee.org/document/8003296

4. Manoj D. "Density Based Traffic Control System" electrical \& electronics engineering Department mahatma Gandhi institute of technology Chaitanya Bharathi P.O., Gandipet, Hyderabad - 5000752012

5. Agarwal Tushar, Singh Akansha and Lakshita " Intelligent Traffic Control Unit", Department of Electronics and Communication Engineering, Bharati Vidyapeeth "s College of Engineering, Paschim Vihar, NewDelhi-110063

6. https://www.iitk.ac.in/nerd/web/artciles/adap tivetraffic-light-timer-control-atltc/

7. Rijurekhasen, Cross Andrew, vashistha Aditya, Venkata N. Padmanabhan, Cutrell Edward, and Thies William "Accurate Speed and Density Measurement for Road Traffic in India" IIT Bombay.

8. Morarescu, Nancy University France, (2011) "Highway traffic model-based density estimation- IEEE Paper" published in American Control Conference (ACC).

9. Bhate, S. V., Kulkarni, P. V., Lagad, S. D., Shinde, M. D., \& Patil, S. (2018, April). "IoT based Intelligent Traffic Signal System for Emergency vehicles". In 2018 Second International Conference on Inventive Communication and Computational Technologies (ICICCT) (pp. 788- 793). IEEE.

10. ang, Bo, Rencheng Zheng, Keisuke Shimono, Tsutomu Kaizuka, and Kimihiko Nakano. (2017) "Evaluation of the effects of in-vehicle traffic lights on driving performances for unsignalised intersections." IET Intelligent Transport Systems 11, no. 2.

11. Higaki, H., (2014, March.) "Virtual Traffic Signals by Cooperation among Vehicle-Mounted Mobile Computers". In New Technologies, Mobility and Security (NTMS), 6th International Conference on (pp. 1-6). IEEE.

12. Rachmadi M. F. et al (2011)., "Adaptive traffic signal control system using camera sensor and embedded system," TENCON 2011 - 2011 IEEE Region 10 Conference, Bali. 